\title{
Stereoselective Synthesis of 4-0-Tosyltetrahydropyrans via Prins Cyclization Reaction of Enol Ethers
}

\author{
Sujit Sarkar \\ Namita Devi \\ Bikoshita Porashar \\ Santu Ruidas
}

Anil K Saikia*

Department of Chemistry, Indian Institute of Technology

Guwahati, Guwahati 781039, Assam, India

asaikia@iitg.ac.in
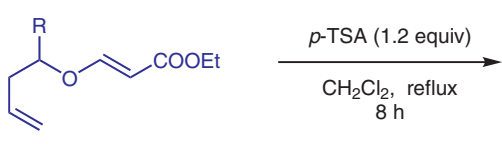

$\mathrm{R}=\mathrm{H}$, alkyl, aryl

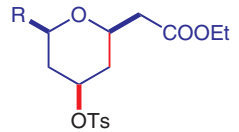

up to $89 \%$ yield 15 examples
Received: 20.01.2019

Accepted after revision: 05.02.2019

Published online: 28.02 .2019

DOI: 10.1055/s-0037-1611679; Art ID: so-2018-d0065-op

License terms: $(c)(1) \ominus(-)$

Abstract Intramolecular cyclization of enol ethers mediated by paratoluenesulfonic acid leads to substituted tetrahydropyrans in good to moderate yields. The reaction is diastereoselective.

Key words tosyl tetrahydropyrans, Prins reaction, enol ether, cyclization, heterocycle

Saturated six-membered cyclic ethers, better known as tetrahydropyrans (THPs), are ubiquitous in nature and represent useful precursors for the synthesis of many biologically active molecules. ${ }^{1}$ For example, neopeltolide $\mathbf{1}$ is a marine natural product isolated from Neopeltidae, collected from the north Jamaican coast. It inhibits cancer cell lines such as A-549 human lung adenocarcinoma, human ovarian sarcoma, and murine leukaemia, with $\mathrm{IC}_{50}$ of $1.2,5.1$, and $0.56 \mathrm{nM}$, respectively. It also inhibits the growth of the fungal pathogen Candida albicans with an inhibitory concentration of $0.62 \mu \mathrm{g} \mathrm{mL} \mathrm{mL}^{-1}$ 4-Hydroxytetrahydropyrancontaining natural products, catechols 2 and 3, isolated from extracts of Plectranthus sylvestris (labiatae), a plant found in the woody hills in East Africa, are potent antioxidants and possess anti-inflammatory properties. ${ }^{3}$ Similarly, apicularen A 4, isolated from various strains of the myxobacterial genus Chondromycesand shows antiproliferative properties against cancer cell lines such as ovarian, prostate, lung, kidney, cervix, leukaemia, and histiocytic cells with $\mathrm{IC}_{50}$ values $0.23-6.79 \mu \mathrm{M}$. It is a novel antiangiogenic agent and may suppress growth of tumors in a dosedependent manner (Figure 1$) .4$

Over the years, the 4-substituted tetrahydropyran rings have been constructed via Lewis or Brønsted acid catalyzed/mediated Prins reaction of homoallyl alcohols and aldehydes followed by trapping with various nucleophiles such as hydroxide, ${ }^{5}$ halide, ${ }^{6}$ aryl, ${ }^{7}$ tosyl, ${ }^{8}$ and nitrile ${ }^{9}$ groups. An earlier report has described the synthesis of 4-hydroxytetrahydropyrans via hydroxyl-Prins cyclization of homoallyl acryloyl ethers mediated by TFA and $\mathrm{K}_{2} \mathrm{CO}_{3} \cdot{ }^{10}$

We have reported the stereoselective synthesis of dihydropyrans, tetrahydrofurans, and tetrahydrothiophenes via Prins reaction of homoallyl, homopropargyl alcohols and thiols. ${ }^{11}$ We have also studied the dual role of $p$-TSA as an activator as well as a nucleophile in the aza-Prins cyclization reaction. ${ }^{12}$ Based on our experience in Prins cyclization<smiles>CCCC(C[C@@H](CC)CC1C[C@@H](OC(=O)/C=C\CCc2coc(/C=C\CNC(=O)OC)n2)C[C@@H](CC(C)C[C@@H](CC)OC(=O)CC)O1)OC</smiles>

Neopeltolide (1)<smiles>CC/C=C\C=C/C(=O)N/C=C/C[C@@H](C[C@@H]1C[C@@H](O)C[C@@H](Cc2cccc(O)c2C(=O)O)O1)OC1CC(O)CC(c2ccc(O)c(O)c2)O1</smiles>

Apicularen A (4)

Figure 1 Some biologically active tetrahydropyrans 
reactions, we envisaged that $p$-TSA could act as a nucleophile and activator in a Prins cyclization reaction of acryloyl ethers. Herein, we wish to report a mild and efficient approach for the synthesis of 4-0-tosyl tetrahydropyrans via Prins reaction of acryloyl enol ethers mediated by $p$-toluenesulfonic acid ( $p$-TSA), which acts as an activator as well as a nucleophile.

Initially, we reacted (E)-ethyl 3-(but-3-en-1-yloxy)acrylate 5a with 1.2 equivalents of $p$-TSA acid in dichloromethane at reflux, and the reaction proceeded smoothly to af-

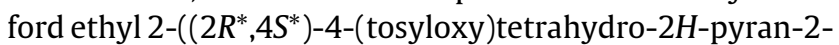
yl)acetate (6a) in $68 \%$ yield as a mixture of 2,4,6-cis and 2,4and 4,6-trans isomers in a ratio of 93:7, with the stereoisomer having the all-cis relationship being the major component. Catalytic amounts of $p$-TSA were found to be inefficient, and excess $p$-TSA (more than 1.2 equiv.) produced a mixture of products. It was also found that only $\mathrm{CH}_{2} \mathrm{Cl}_{2}$ is a suitable solvent; other polar protic solvents such as $\mathrm{CH}_{3} \mathrm{OH}$, EtOH, $\mathrm{CH}_{3} \mathrm{COOH}$ and polar aprotic solvents such as $\mathrm{CH}_{3} \mathrm{CN}$, THF, DMF, DMSO did not result in the desired product.

To investigate the substrate scope with these optimal reaction conditions, a variety of homoallyl acryloyl ethers was synthesized according to reported methods by Michael addition of homoallyl alcohols to ethyl propiolate using $\mathrm{N}$ methyl morpholine (NMM) as a base in dichloromethane at ambient temperature. (Scheme 1$).{ }^{11 \mathrm{~b}}$

These acryloyl enol ethers were subjected to $p$-TSA-mediated Prins cyclization; the results are shown in Table 1. Both aliphatic and aromatic substituted acryloyl ethers worked well and produced moderate to high yields of cyclized material with high diastereoselectivity. Substrates having aromatic substituents (entries 2-6, 9-12, 16) gave higher yields compared with aliphatic substrates (entries 8 , 13-14 and 17). Substrates having aromatic substituents with highly electron-donating (entry 7) or electron-withdrawing groups (entry 15) did not furnish the desired products. This might be due to the interaction of the acid with methoxy ${ }^{13}$ and nitro ${ }^{14}$ groups of the aromatic aldehydes.

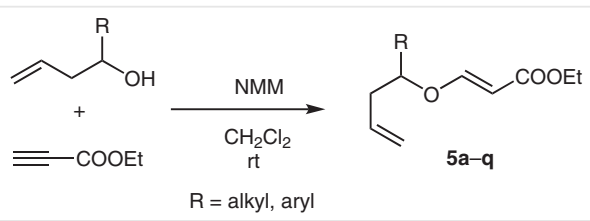

Scheme 1 Synthesis of starting materials

The reaction is highly diastereoselective, with a cis-relationship among the substituents on the tetrahydropyran ring. The diastereoselectivity was confirmed by ${ }^{1} \mathrm{H}$ and ${ }^{13} \mathrm{C}$ NMR spectroscopic analysis of the crude product, and the cis stereochemistry was determined based on NOE analysis of compound $\mathbf{6 f}$. A strong NOE between hydrogen at C-2 and C-4 as well as interaction between C-2 and C-6 clearly suggests all substituents are in a cis relationship (Scheme 2).

Table 1 Synthesis of O-Tosylated Tetrahydropyrans via Prins Cyclization Reaction

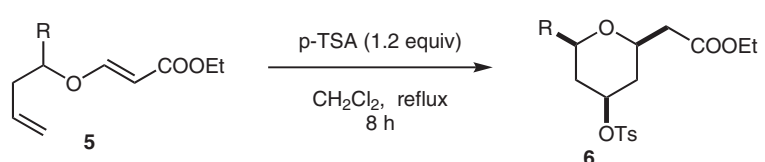

\begin{tabular}{|c|c|c|c|c|}
\hline Entry & 5 & 6 & Ratio $^{a}$ & Yield (\%) \\
\hline 1 & & & $93: 7$ & 68 \\
\hline 2 & & & $93: 7$ & 74 \\
\hline 3 & & & 99:1 & 79 \\
\hline
\end{tabular}


38

THIEME

SynOpen

S. Sarkar et al.

OPEN
ACCESS

Paper

Table 1 (continued)

Entry $\mathbf{5}$

4

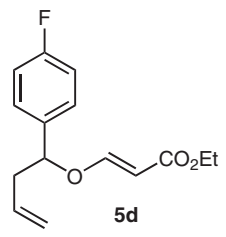

5

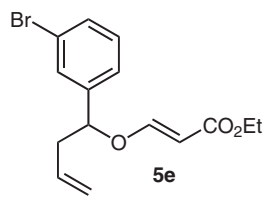

6

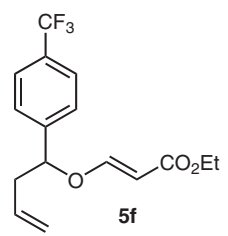

7

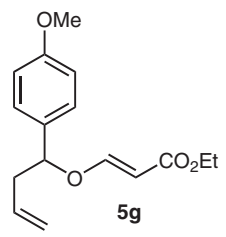

8

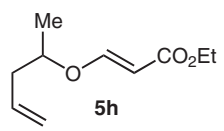

9<smiles>C=CCC(O/C=C/C(=O)OCC)c1ccc(Br)cc1</smiles>

10

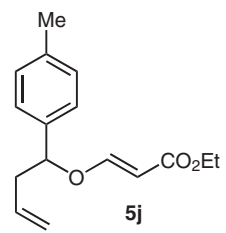

11
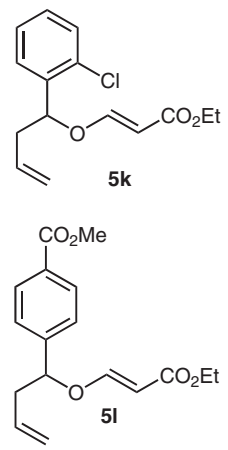

6

Ratio $^{\mathrm{a}}$

Yield (\%) ${ }^{\mathrm{b}}$

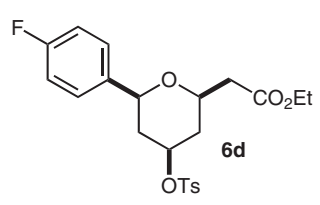

95:5

76

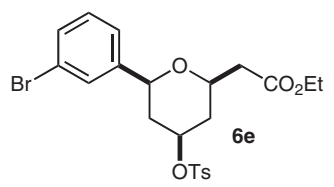

95:5

84

$98: 2$

89

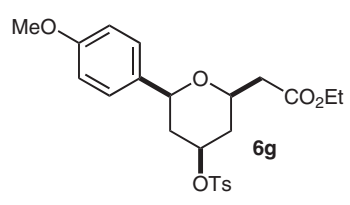

$\underbrace{\mathrm{Meh}}_{\mathrm{OTS}_{\mathrm{T}}} \mathrm{CO}_{2} \mathrm{Et}$

$86: 14$

74

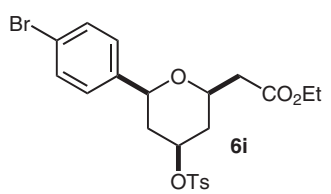

$92: 8$

75

$96: 4$

62

$92: 8$

72

$96: 4$

64

Georg Thieme Verlag Stuttgart · New York - SynOpen 2019, 3, 36-45 
Table 1 (continued)

\begin{tabular}{|c|c|c|c|c|}
\hline Entry & 5 & 6 & Ratio $^{a}$ & Yield (\%) \\
\hline 13 & & & $82: 18$ & 54 \\
\hline 14 & & & $93: 7$ & 52 \\
\hline 15 & & & - & 0 \\
\hline 16 & & & $90: 10$ & 64 \\
\hline 17 & & & 100:0 & 54 \\
\hline
\end{tabular}

a Ratio determined by ${ }^{1} \mathrm{H}$ NMR analysis.

${ }^{\mathrm{b}}$ Yield refers to isolated yield.

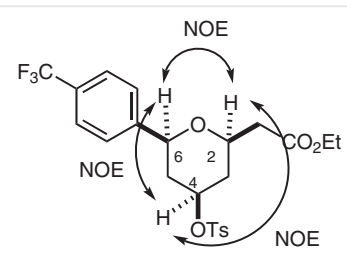

Scheme 2 Key NOE enhancements of $\left(2 R^{*}, 4 S^{*}, 6 S^{*}\right)$-ethyl 4-(tosyloxy)-6-(4-(trifluoromethyl)phenyl)tetrahydro-2H-pyran-2-carboxylate (6f)

A mechanism proposed for the diastereoselectivity of the reaction involves $p$-TSA protonating the carbonyl group of the ester, leading to formation of oxocarbenium ion $\mathbf{B}$, which, after Prins cyclization, forms tetrahydropyranyl cation $\mathbf{C}$ (Scheme 3). The tosyl nucleophile attacks carbocation C equatorially, to give $\mathbf{6}$ as the major product and $\mathbf{7}$ as the minor product. This might be due to the lower steric repulsion between the $\mathrm{C}-2 \mathrm{H}$ and $\mathrm{C}-4 \mathrm{H}$ hydrogens compared with intermediate $\mathbf{E}$, where there is a strong steric repulsion between the $\mathrm{C}-2 \mathrm{H}$ hydrogen and the $\mathrm{C}-4$ tosyl group.

This methodology was further extended to the synthesis of 4-iodotetrahydropyrans (Scheme 4). Thus, the reaction of 4-tosyl tetrahydropyrans $\mathbf{6 a}, \mathbf{6 c}$, and $\mathbf{6 e}$ with cerium(III) chloride heptahydrate and sodium iodide in acetonitrile at $85{ }^{\circ} \mathrm{C}$ gave the corresponding 4-iodotetrahydropyrans 8a, 8c, and $\mathbf{8 e}$ in moderate yields. 2,4-Disubstituted tosyltetrahydropyran $\mathbf{6 a}$ gave a single diastereomer 8a (55\%); whereas 2,4,6-trisubstituted tosyltetrahydropyrans $\mathbf{6 c}(58 \%)$ and $\mathbf{6 e}(55 \%)$ gave inseparable mixture of diastereomers $\mathbf{8 c}$ and $\mathbf{8 e}$ with a ratio of $65: 35$ and $67: 33$, respectively. 

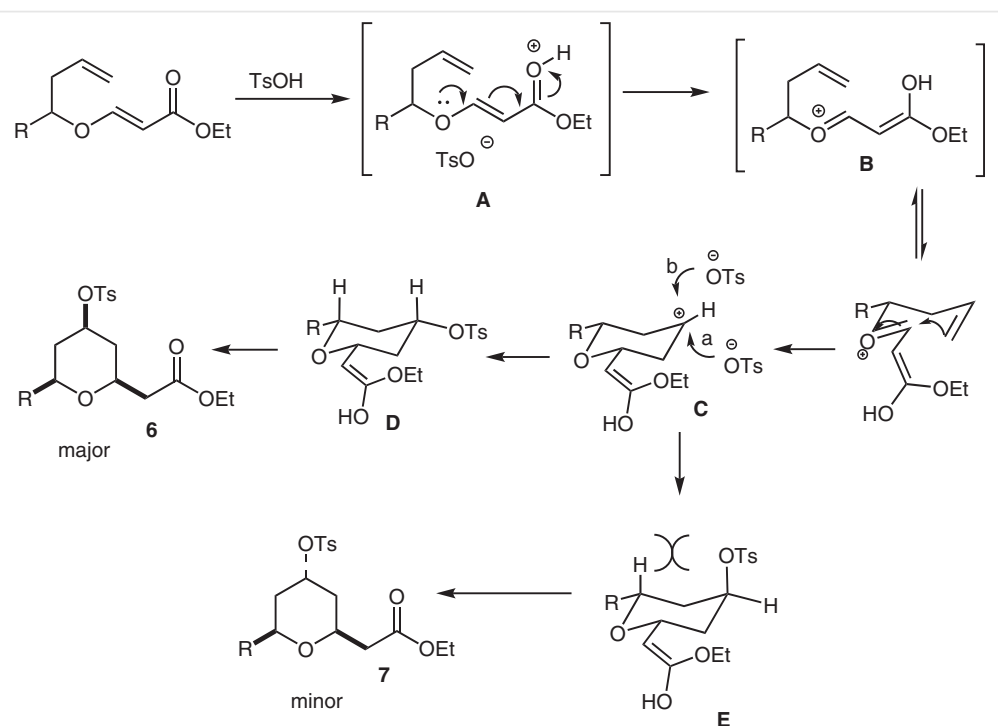

Scheme 3 Plausible reaction mechanism

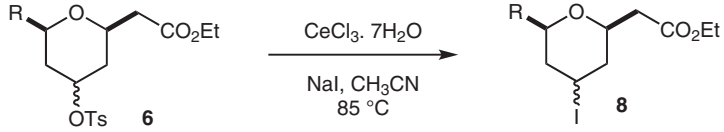

$\mathrm{R}=\mathrm{H}$, aryl

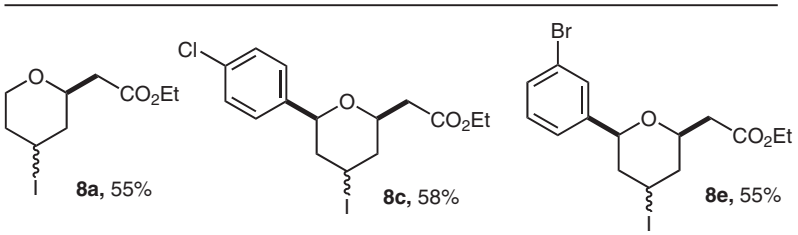

Scheme 4 Synthesis of 4-iodotetrahydropyran

Similarly, the tosyl group of the 4-tosyl tetrahydropyrans $\mathbf{6 a}, \mathbf{6 c}$, and $\mathbf{6 p}$ can be reduced by sodium borohydride in dimethylsulfoxide at $85{ }^{\circ} \mathrm{C}$ to give 9a, 9c, and $\mathbf{9 p}$ in 53 , 68 , and $70 \%$ yields, respectively (Scheme 5 ).
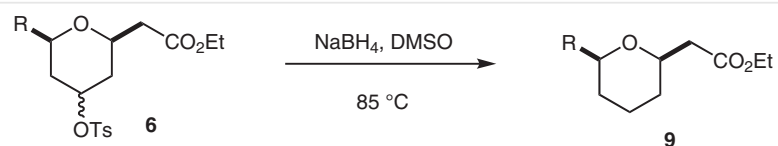

$\mathrm{R}=\mathrm{H}$, ary

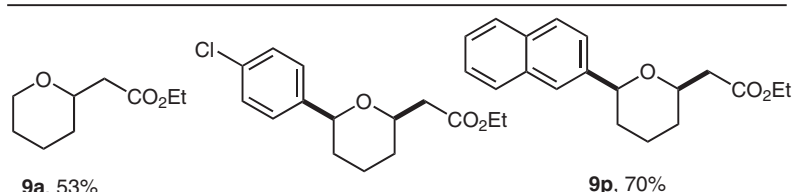

$9 a, 53 \%$

$9 c, 68 \%$

$9 p, 70 \%$

Scheme 5 Detosylation of 4-tosyltetrahydropyran

The tosyl group of the 4-tosyl tetrahydropyran can also be substituted with an azide group (Scheme 6). Compounds 6c and 6h, when treated with sodium azide in DMF at
$100{ }^{\circ} \mathrm{C}$, provided the corresponding azido compounds $10 \mathrm{c}$ and $\mathbf{1 0 h}$ in 66 and $60 \%$ yields, respectively, with inversion of configuration at $\mathrm{C}-4$. The configuration of the compounds was determined by NOE analysis of compound 10c. It is noteworthy that the reaction gave only a single diastereomer.

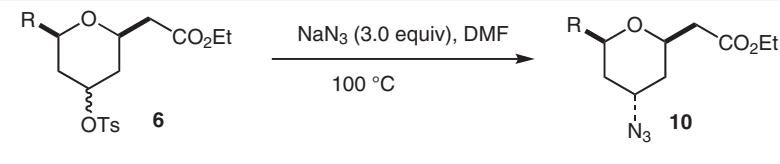

$$
\mathrm{R}=\text { aryl }
$$
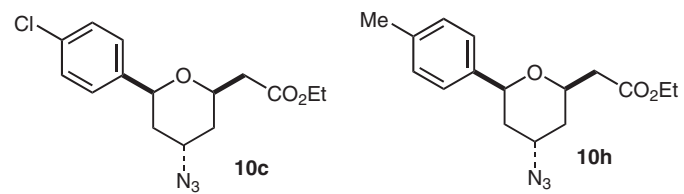

Scheme 6 Synthesis of 4-azidotetrahydropyrans

In conclusion, a methodology for the stereoselective synthesis of tosyl substituted tetrahydropyrans through Prins cyclization reaction of acryloyl enol ethers has been developed. Good yields are achieved with high diastereoselectivity. This methodology will be useful for the synthesis of other substituted pyran rings such as 4-iodotetrahydropyrans and 2,6-disubstituted tetrahydropyrans by substitution or reduction of the tosyl functionality, respectively. The tosyl group can also be replaced by an azido group to prepare 4-azidotetrahydropyrans with inversion of configuration at the 4-position. 
All the reagents were of reagent grade (AR grade) and were used as purchased without further purification. Silica gel (60-120 mesh size) was used for column chromatography. Reactions were monitored by TLC on silica gel $\mathrm{GF}_{254}(0.25 \mathrm{~mm})$. Melting points were determined with a Büchi B-540 melting point apparatus. IR spectra were recorded with a Fourier transform infrared (FTIR) spectrometer either as a neat liquid or as $\mathrm{KBr}$ pellets. NMR spectra were obtained with $400 \mathrm{MHz}$ $\left({ }^{1} \mathrm{H}, 400 \mathrm{MHz}\right.$ and $\left.{ }^{13} \mathrm{C}, 100 \mathrm{MHz}\right)$ and $600 \mathrm{MHz}\left({ }^{1} \mathrm{H}, 600 \mathrm{MHz}\right.$ and ${ }^{13} \mathrm{C}$, $150 \mathrm{MHz}$ ) instruments using $\mathrm{CDCl}_{3}$ as solvent and tetramethylsilane as internal standard. Chemical shifts $(\delta)$ are reported in parts per million (ppm) and coupling constants $(J)$ are given in $\mathrm{Hz}$. High-resolution mass spectra (HRMS) were measured with a Q-TOF mass analyzer.

\section{Synthesis of Starting Materials}

The homoallyl acryloyl ethers were synthesized by following the reported procedure and the structures and purities of known compounds $\mathbf{5 a}-\mathbf{q}$ were confirmed by comparison of their spectroscopic data $\left({ }^{1} \mathrm{H}\right.$ NMR and ${ }^{13} \mathrm{C}$ NMR $)$ with reported data. ${ }^{11 b}$

\section{Synthesis of 4-Tosyloxy Tetrahydropyrans; Typical Procedure}

To (E)-ethyl 3-(but-3-en-1-yloxy) acrylate (340 mg, $0.5 \mathrm{mmol}$ ) in dichloromethane $(3 \mathrm{~mL})$ was added $p$-toluenesulfonic acid monohydrate $(408 \mathrm{mg}, 0.6 \mathrm{mmol})$. The reaction mixture was heated to reflux for $8 \mathrm{~h}$ and the progress of the reaction was monitored by TLC (EtO$\mathrm{Ac} /$ hexane, 1:4). Upon completion, the reaction was quenched with aq $\mathrm{NaHCO}_{3}$, extracted (EtOAc), washed with brine, dried $\left(\mathrm{Na}_{2} \mathrm{SO}_{4}\right)$, filtered, and evaporated under reduced pressure. The crude product was purified by column chromatography over silica gel, eluting with EtO-

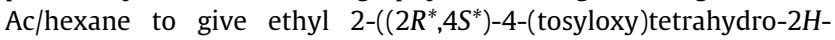
pyran-2-yl)acetate (6a) in $68 \%$ yield.

\section{Ethyl 2-((2R* $\left.4 S^{*}\right)-4$-(Tosyloxy)tetrahydro-2H-pyran-2-yl)acetate} (6a, Diastereomeric Mixture, 93:7)

Yield: $112 \mathrm{mg}$ (68\%); pale-yellow gum.

${ }^{1} \mathrm{H}$ NMR $\left(600 \mathrm{MHz}, \mathrm{CDCl}_{3}\right): \delta=1.24(\mathrm{t}, J=7.2 \mathrm{~Hz}, 3 \mathrm{H}), 1.46-1.52(\mathrm{~m}$, $1 \mathrm{H}), 1.67-1.75(\mathrm{~m}, 2 \mathrm{H}), 1.84-1.88(\mathrm{~m}, 1 \mathrm{H}), 1.97-2.00(\mathrm{~m}, 1 \mathrm{H}), 2.36$ (dd, $J=15.6,5.4 \mathrm{~Hz}, 1 \mathrm{H}), 2.45(\mathrm{~s}, 3 \mathrm{H}), 2.52(\mathrm{dd}, J=15.6,8.4 \mathrm{~Hz}, 1 \mathrm{H})$, $3.36(\mathrm{dd}, J=12.0,1.2 \mathrm{~Hz}, 1 \mathrm{H}), 3.69-3.75(\mathrm{~m}, 1 \mathrm{H}), 3.96(\mathrm{dd}, J=12.0$, $4.2 \mathrm{~Hz}, 1 \mathrm{H}), 4.13(\mathrm{q}, J=7.2 \mathrm{~Hz}, 2 \mathrm{H}), 4.56-4.62(\mathrm{~m}, 1 \mathrm{H}), 7.35(\mathrm{~d}, J=$ $7.8 \mathrm{~Hz}, 2 \mathrm{H}), 7.79(\mathrm{~d}, J=7.8 \mathrm{~Hz}, 2 \mathrm{H})$.

${ }^{13} \mathrm{C} \mathrm{NMR}\left(150 \mathrm{MHz}, \mathrm{CDCl}_{3}\right): \delta=14.3,21.8,32.6,38.1,41.1,60.9,65.6$, 72.6, 77.9, 127.8, 130.1, 134.5, 145.0, 170.7 .

IR (KBr, neat): 2968, 2863, 1738, 1598, 1362, 1175, 1083, 899, 670, $576 \mathrm{~cm}^{-1}$.

HRMS (ESI): $m / z[M+H]^{+}$calcd. for $\mathrm{C}_{16} \mathrm{H}_{23} \mathrm{O}_{6} \mathrm{~S}$ : 343.1210; found: 343.1239.

Ethyl 2-((2R* $\left.4 R^{*}, 6 S^{*}\right)-6$-Phenyl-4-(tosyloxy)tetrahydro-2H-pyran2-yl)acetate (6b, Diastereomeric Mixture, 93:7)

Yield: $150 \mathrm{mg}$ (74\%); white solid, $\mathrm{mp} 87-89^{\circ} \mathrm{C}$.

${ }^{1} \mathrm{H}$ NMR $\left(600 \mathrm{MHz}, \mathrm{CDCl}_{3}\right): \delta=1.22(\mathrm{t}, J=7.2 \mathrm{~Hz}, 3 \mathrm{H}), 1.55-1.60(\mathrm{~m}$, $1 \mathrm{H}), 1.68-1.74(\mathrm{~m}, 1 \mathrm{H}), 2.06-2.10(\mathrm{~m}, 1 \mathrm{H}), 2.21-2.24(\mathrm{~m}, 1 \mathrm{H}), 2.45$ $(\mathrm{s}, 3 \mathrm{H}), 2.46$ (dd, $J=15.6,6.0 \mathrm{~Hz}, 1 \mathrm{H}), 2.65(\mathrm{dd}, J=15.6,7.8 \mathrm{~Hz}, 1 \mathrm{H})$, 3.92-3.96 (m, $1 \mathrm{H}), 4.12(\mathrm{q}, J=7.2 \mathrm{~Hz}, 2 \mathrm{H}), 4.37$ (d, $J=10.8 \mathrm{~Hz}, 1 \mathrm{H})$, 4.75-4.80 (m, $1 \mathrm{H}), 7.26-7.36(\mathrm{~m}, 7 \mathrm{H}), 7.81(\mathrm{~d}, J=7.8 \mathrm{~Hz}, 2 \mathrm{H})$.
${ }^{13} \mathrm{C}$ NMR $\left(150 \mathrm{MHz}, \mathrm{CDCl}_{3}\right): \delta=14.3,21.8,37.6,39.6,40.8,41.0,41.1$, $60.8,60.9,72.3,78.0,126.0,127.8,127.9,128.0,128.5,128.6,130.1$, $134.4,140.8,145.0,170.7$

IR (KBr, neat): 2873, 1735, 1598, 1413, 1366, 1176, 1097, 956, 758, $669,579 \mathrm{~cm}^{-1}$

HRMS (ESI): $m / z[M+H]^{+}$calcd. for $\mathrm{C}_{22} \mathrm{H}_{27} \mathrm{O}_{6} \mathrm{~S}: 419.1536$; found: 419.1536.

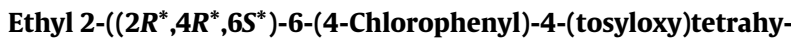
dro-2H-pyran-2-yl)acetate (6c, Diastereomeric Mixture, 99:1)

Yield: $173 \mathrm{mg}$ (79\%); pale-yellow gum.

${ }^{1} \mathrm{H}$ NMR $\left(600 \mathrm{MHz}, \mathrm{CDCl}_{3}\right): \delta=1.22(\mathrm{t}, J=7.2 \mathrm{~Hz}, 3 \mathrm{H}), 1.52-1.59(\mathrm{~m}$, $1 \mathrm{H}), 1.62-1.68(\mathrm{~m}, 1 \mathrm{H}), 2.06-2.09(\mathrm{~m}, 1 \mathrm{H}), 2.20-2.23(\mathrm{~m}, 1 \mathrm{H}), 2.45$ $(\mathrm{s}, 3 \mathrm{H}), 2.46(\mathrm{dd}, J=15.6,5.4 \mathrm{~Hz}, 1 \mathrm{H}), 2.63(\mathrm{dd}, J=15.6,7.2 \mathrm{~Hz}, 1 \mathrm{H})$, 3.92-3.94 (m, $1 \mathrm{H}), 4.11(\mathrm{q}, J=7.2 \mathrm{~Hz}, 2 \mathrm{H}), 3.35$ (dd, $J=11.4,1.8 \mathrm{~Hz}$, $1 \mathrm{H}), 4.75-4.79(\mathrm{~m}, 1 \mathrm{H}), 7.20(\mathrm{~d}, J=8.4 \mathrm{~Hz}, 2 \mathrm{H}), 7.28(\mathrm{~d}, J=8.4 \mathrm{~Hz}$, $2 \mathrm{H}), 7.35$ (d, $J=8.4 \mathrm{~Hz}, 2 \mathrm{H}), 7.80(\mathrm{~d}, J=8.4 \mathrm{~Hz}, 2 \mathrm{H})$.

${ }^{13} \mathrm{C}$ NMR $\left(150 \mathrm{MHz}, \mathrm{CDCl}_{3}\right): \delta=14.3,21.9,37.5,39.7,41.0,60.9,72.4$, 76.5, 77.6, 127.3, 127.8, 128.7, 130.1, 133.7, 134.3, 139.4, 145.1, 170.6.

IR (KBr, neat): 2927, 2854, 1738, 1599, 1494, 1189, 1090, 956, 815, $757,667,555 \mathrm{~cm}^{-1}$.

HRMS (ESI): $m / z[\mathrm{M}+\mathrm{H}]^{+}$calcd. for $\mathrm{C}_{22} \mathrm{H}_{26} \mathrm{ClO}_{5} \mathrm{~S}: 453.1133$; found: 453.1123.

Ethyl 2-((2R* $\left.4 R^{*}, 6 S^{*}\right)-6$-(4-Fluorophenyl)-4-(tosyloxy)tetrahydro2H-pyran-2-yl)acetate (6d, Diastereomeric Mixture, 95:5)

Yield: $160 \mathrm{mg}$ (76\%); pale-yellow gum.

${ }^{1} \mathrm{H}$ NMR $\left(600 \mathrm{MHz}, \mathrm{CDCl}_{3}\right): \delta=1.22(\mathrm{t}, J=7.2 \mathrm{~Hz}, 3 \mathrm{H}), 1.53-1.56(\mathrm{~m}$, $1 \mathrm{H}), 1.65-1.71(\mathrm{~m}, 1 \mathrm{H}), 2.05-2.09(\mathrm{~m}, 1 \mathrm{H}), 2.21-2.24(\mathrm{~m}, 1 \mathrm{H}), 2.45$ $(\mathrm{dd}, J=15.6,5.4 \mathrm{~Hz}, 1 \mathrm{H}), 2.46(\mathrm{~s}, 3 \mathrm{H}), 2.64(\mathrm{dd}, J=15.6,7.2 \mathrm{~Hz}, 1 \mathrm{H})$, 3.91-3.95 (m, $1 \mathrm{H}), 4.12(\mathrm{q}, J=7.2 \mathrm{~Hz}, 2 \mathrm{H}), 4.35(\mathrm{dd}, J=11.4,1.8 \mathrm{~Hz}$, $1 \mathrm{H}), 4.74-4.80(\mathrm{~m}, 1 \mathrm{H}), 7.00(\mathrm{t}, J=8.4 \mathrm{~Hz}, 2 \mathrm{H}), 7.22-7.25(\mathrm{~m}, 2 \mathrm{H})$, $7.35(\mathrm{~d}, J=8.4 \mathrm{~Hz}, 2 \mathrm{H}), 7.80(\mathrm{~d}, J=8.4 \mathrm{~Hz}, 2 \mathrm{H})$.

${ }^{13} \mathrm{C} \mathrm{NMR}\left(150 \mathrm{MHz}, \mathrm{CDCl}_{3}\right): \delta=14.4,21.9,37.6,39.7,41.0,60.9,72.4$, 76.6, 77.8, $115.4(\mathrm{~d}, J=19.5 \mathrm{~Hz}), 127.6(\mathrm{~d}, J=7.5 \mathrm{~Hz}), 127.9,130.1$, $134.4,136.7,145.1,162.5(\mathrm{~d}, J=246.0 \mathrm{~Hz}), 170.6$.

IR (KBr, neat): 2928, 1733, 1599, 1367, 1176, 1085, 768, 668, $551 \mathrm{~cm}^{-1}$. HRMS (ESI): $m / z$ [M $+\mathrm{H}]^{+}$calcd. for $\mathrm{C}_{22} \mathrm{H}_{26} \mathrm{FO}_{6} \mathrm{~S}: 437.1429$; found: 437.1435.

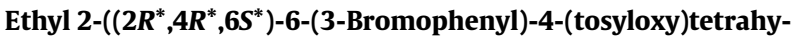
dro-2H-pyran-2-yl)acetate (6e, Diastereomeric Mixture, 95:5)

Yield: $202 \mathrm{mg}$ (84\%); pale-yellow gum.

${ }^{1} \mathrm{H}$ NMR $\left(600 \mathrm{MHz}, \mathrm{CDCl}_{3}\right): \delta=1.22(\mathrm{t}, J=7.2 \mathrm{~Hz}, 3 \mathrm{H}), 1.41-1.47(\mathrm{~m}$, $1 \mathrm{H}), 1.56-1.62(\mathrm{~m}, 1 \mathrm{H}), 2.12-2.16(\mathrm{~m}, 1 \mathrm{H}), 2.24-2.28(\mathrm{~m}, 1 \mathrm{H}), 2.45$ $(\mathrm{s}, 3 \mathrm{H}), 2.49(\mathrm{dd}, J=15.6,5.4 \mathrm{~Hz}, 1 \mathrm{H}), 2.65(\mathrm{dd}, J=15.6,7.8 \mathrm{~Hz}, 1 \mathrm{H})$, $3.96-4.00(\mathrm{~m}, 1 \mathrm{H}), 4.12(\mathrm{q}, J=7.2 \mathrm{~Hz}, 2 \mathrm{H}), 4.60(\mathrm{dd}, J=11.4,1.8 \mathrm{~Hz}$, $1 \mathrm{H}), 4.75-4.81(\mathrm{~m}, 1 \mathrm{H}), 7.09-7.13(\mathrm{~m}, 1 \mathrm{H}), 7.29(\mathrm{t}, J=7.2 \mathrm{~Hz}, 1 \mathrm{H})$, $7.35(\mathrm{~d}, J=8.4 \mathrm{~Hz}, 2 \mathrm{H}), 7.44-7.47(\mathrm{~m}, 2 \mathrm{H}), 7.81(\mathrm{~d}, J=8.4 \mathrm{~Hz}, 2 \mathrm{H})$.

${ }^{13} \mathrm{C}$ NMR $\left(150 \mathrm{MHz}, \mathrm{CDCl}_{3}\right): \delta=14.4,21.9,37.7,38.2,41.2,60.9,72.5$, 76.4, 77.7, 121.6, 127.6, 127.9, 129.3, 130.2, 132.7, 134.4, 140.1, 145.1, 170.5 .

IR (KBr, neat): 2980, 2929, 1735, 1598, 1495, 1366, 1177, 1078, 957 , $815,770,667,556 \mathrm{~cm}^{-1}$.

HRMS (ESI): $m / z$ [M + H] $]^{+}$calcd. for $\mathrm{C}_{22} \mathrm{H}_{26} \mathrm{Br}^{81} \mathrm{O}_{6} \mathrm{~S}$ : 499.0608; found: 499.0628 . 


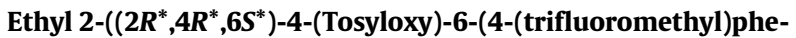
nyl)-tetrahydro-2H-pyran-2-yl)acetate (6f, Diastereomeric Mixture, 98:2)

Yield: $210 \mathrm{mg}$ (89\%); white solid; $\mathrm{mp} 120-122^{\circ} \mathrm{C}$.

${ }^{1} \mathrm{H} \mathrm{NMR}\left(600 \mathrm{MHz}, \mathrm{CDCl}_{3}\right): \delta=1.23(\mathrm{t}, J=7.2 \mathrm{~Hz}, 3 \mathrm{H}), 1.55-1.61(\mathrm{~m}$, $1 \mathrm{H}), 1.62-1.69$ ( $\mathrm{m}, 1 \mathrm{H}), 2.07-2.10(\mathrm{~m}, 1 \mathrm{H}), 2.25-2.29(\mathrm{~m}, 1 \mathrm{H}), 2.46$ (s, $3 \mathrm{H}), 2.47$ (dd, $J=15.6,5.4 \mathrm{~Hz}, 1 \mathrm{H}), 2.65$ (dd, $J=15.6,7.8 \mathrm{~Hz}, 1 \mathrm{H}$ ), $3.94-3.68(\mathrm{~m}, 1 \mathrm{H}), 4.12(\mathrm{q}, J=7.2 \mathrm{~Hz}, 2 \mathrm{H}), 4.44(\mathrm{dd}, J=11.4,1.8 \mathrm{~Hz}$, $1 \mathrm{H}), 4.77-4.82(\mathrm{~m}, 1 \mathrm{H}), 7.35(\mathrm{~d}, J=8.4 \mathrm{~Hz}, 2 \mathrm{H}), 7.39(\mathrm{~d}, J=8.4 \mathrm{~Hz}$, $2 \mathrm{H}), 7.57(\mathrm{~d}, J=8.4 \mathrm{~Hz}, 2 \mathrm{H}), 7.57(\mathrm{~d}, J=8.4 \mathrm{~Hz}, 2 \mathrm{H})$.

${ }^{13} \mathrm{C}$ NMR $\left(150 \mathrm{MHz}, \mathrm{CDCl}_{3}\right): \delta=14.4,21.9,37.5,39.8,41.0,60.9,72.5$, $76.5,77.5,125.5,126.2,126.8(\mathrm{q}, J=270.0 \mathrm{~Hz}), 127.9,130.1,130.2(\mathrm{q}$, $J=36 \mathrm{~Hz}), 134.3,144.8,145.2,170.6$.

IR (KBr, neat): 2930, 2860, 1735, 1622, 1599, 1370, 1163, 1018, 841, $759,671,555 \mathrm{~cm}^{-1}$.

HRMS (ESI): $m / z[\mathrm{M}+\mathrm{H}]^{+}$calcd. for $\mathrm{C}_{23} \mathrm{H}_{26} \mathrm{~F}_{3} \mathrm{O}_{6} \mathrm{~S}$ : 487.1397; found: 487.1388.

\section{Ethyl 2-((2R* $\left.4 S^{*}, 6 R^{*}\right)-6-M e t h y l-4-(t o s y l o x y) t e t r a h y d r o-2 H-$} pyran-2-yl)acetate (6h, Diastereomeric Mixture; 86:16)

Yield: $263 \mathrm{mg}$ (74\%); yellow gum.

${ }^{1} \mathrm{H} \mathrm{NMR}\left(400 \mathrm{MHz}, \mathrm{CDCl}_{3}\right): \delta=1.08(\mathrm{~d}, J=6.2 \mathrm{~Hz}, 3 \mathrm{H}), 1.17(\mathrm{t}, J=7.2$, $3 \mathrm{H}), 1.25-1.37$ (m, $2 \mathrm{H}$ ), 1.84-1.90 (m, $2 \mathrm{H}), 2.28$ (dd, $J=15.4,5.4 \mathrm{~Hz}$, $1 \mathrm{H}), 2.36(\mathrm{~s}, 3 \mathrm{H}), 2.46(\mathrm{dd}, J=15.4,7.6 \mathrm{~Hz}, 1 \mathrm{H}), 3.34-3.36(\mathrm{~m}, 1 \mathrm{H})$, 3.64-3.75 (m, $1 \mathrm{H}), 4.06(\mathrm{q}, J=7.2 \mathrm{~Hz}, 2 \mathrm{H}), 4.48-4.55(\mathrm{~m}, 1 \mathrm{H}), 7.27$ (d, $J=8.0 \mathrm{~Hz}, 2 \mathrm{H}), 7.79$ (d, $J=8.0 \mathrm{~Hz}, 2 \mathrm{H}$ ).

${ }^{13} \mathrm{C}$ NMR $\left(150 \mathrm{MHz}, \mathrm{CDCl}_{3}\right): \delta=14.2,21.4,21.7,37.3,39.5,40.9,60.2$, 71.5, 71.7, 77.9, 127.6, 127.7, 129.9, 134.3, 144.8, 170.6.

IR (KBr, neat): 2961, 2862, 1730, 1594, 1360, 1174, 1081, 894, 671, $572 \mathrm{~cm}^{-1}$.

HRMS (ESI): $m / z[M+H]^{+}$calcd. for $\mathrm{C}_{17} \mathrm{H}_{25} \mathrm{O}_{6} \mathrm{~S}$ : 357.1366; found: 357.1364.

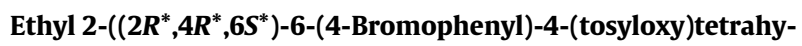
dro-2H-pyran-2-yl)acetate (6i, diastereomeric mixture; 92:8)

Yield: (372 mg, 75\%); reddish oil.

${ }^{1} \mathrm{H} \mathrm{NMR}\left(400 \mathrm{MHz}, \mathrm{CDCl}_{3}\right): \delta=1.24(\mathrm{t}, J=7.2 \mathrm{~Hz}, 3 \mathrm{H}), 1.51-1.69(\mathrm{~m}$, $2 \mathrm{H}), 2.04-2.09(\mathrm{~m}, 1 \mathrm{H}), 2.18-2.26(\mathrm{~m}, 1 \mathrm{H}), 2.46$ (s, $3 \mathrm{H}), 2.47$ (dd, $J=$ 15.0, 7.2 Hz, $1 \mathrm{H}), 2.65(\mathrm{dd}, J=15.0,7.8 \mathrm{~Hz}, 1 \mathrm{H}), 3.90-3.96(\mathrm{~m}, 1 \mathrm{H})$, $4.13(\mathrm{q}, J=7.2 \mathrm{~Hz}, 2 \mathrm{H}), 4.35(\mathrm{~d}, J=10.5 \mathrm{~Hz}, 1 \mathrm{H}), 4.71-4.80(\mathrm{~m}, 1 \mathrm{H})$, $7.18(\mathrm{~d}, J=8.0 \mathrm{~Hz}, 2 \mathrm{H}), 7.35(\mathrm{~d}, J=8.0 \mathrm{~Hz}, 2 \mathrm{H}), 7.41(\mathrm{~d}, J=8.0 \mathrm{~Hz}$, $2 \mathrm{H}), 7.80(\mathrm{~d}, J=8.0 \mathrm{~Hz}, 2 \mathrm{H})$.

${ }^{13} \mathrm{C}$ NMR $\left(150 \mathrm{MHz}, \mathrm{CDCl}_{3}\right): \delta=14.4,21.9,37.5,39.7,41.0,60.9,72.4$, 76.4, 77.6, 122.7, 124.5, 127.9, 129.1, 130.2, 134.3, 143.0, 145.1, 176.8 . IR (KBr, neat): 2932, 2862, 1739, 1628, 1600, 1374, 1169, 1012, 842, $758,679,559 \mathrm{~cm}^{-1}$.

HRMS (ESI): $m / z[M+H]^{+}$calcd. for $\mathrm{C}_{22} \mathrm{H}_{26} \mathrm{BrO}_{6} \mathrm{~S}$ : 497.0628; (Br79); found: 497.0640 .

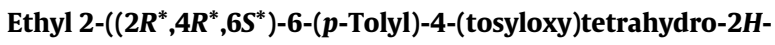
pyran-2-yl)acetate (6j, diastereomeric mixture; 96:4)

Yield: $267 \mathrm{mg}$ (64\%); yellow oil.

${ }^{1} \mathrm{H}$ NMR $\left(600 \mathrm{MHz}, \mathrm{CDCl}_{3}\right): \delta=1.26(\mathrm{t}, J=7.2 \mathrm{~Hz}, 3 \mathrm{H}), 1.51-1.60(\mathrm{~m}$, $1 \mathrm{H}), 1.64-1.75(\mathrm{~m}, 1 \mathrm{H}), 2.10(\mathrm{~d}, J=10.0 \mathrm{~Hz}, 1 \mathrm{H}), 2.22(\mathrm{~d}, J=10.0 \mathrm{~Hz}$, $1 \mathrm{H}), 2.33(\mathrm{~s}, 3 \mathrm{H}), 2.47(\mathrm{~s}, 3 \mathrm{H}), 2.63-2.70(\mathrm{~m}, 1 \mathrm{H}), 3.91-3.95(\mathrm{~m}, 1 \mathrm{H})$, $4.10-4.16(\mathrm{~m}, 2 \mathrm{H}), 4.32-4.38(\mathrm{~m}, 1 \mathrm{H}), 4.75-4.85(\mathrm{~m}, 1 \mathrm{H}), 7.13-7.18$ $(\mathrm{m}, 4 \mathrm{H}), 7.36(\mathrm{~d}, J=8.4 \mathrm{~Hz}, 2 \mathrm{H}), 7.82(\mathrm{~d}, J=8.4 \mathrm{~Hz}, 2 \mathrm{H})$.
${ }^{13} \mathrm{C}$ NMR $\left(150 \mathrm{MHz}, \mathrm{CDCl}_{3}\right): \delta=14.4,21.3,21.9,37.7,39.6,41.1,60.8$, 72.3, 77.0, 78.2, 126.0, 127.8, 129.3, 130.1, 134.4, 137.8, 137.9, 145.0, 170.7.

IR (KBr, neat): 2932, 2865, 1734, 1621, 1594, 1371, 1162, 1012, 842 , $754,672,5556 \mathrm{~cm}^{-1}$.

HRMS (ESI): $m / z[M+H]^{+}$calcd. for $\mathrm{C}_{23} \mathrm{H}_{29} \mathrm{O}_{6} \mathrm{~S}: 433.1679$; found: 433.1693.

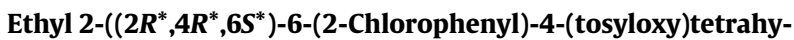
dro-2H-pyran-2-yl)acetate (6k, Diastereomeric Mixture; 92:8) Yield: $326 \mathrm{mg}$ (72\%); yellow oil.

${ }^{1} \mathrm{H}$ NMR $\left(600 \mathrm{MHz}, \mathrm{CDCl}_{3}\right): \delta=1.21(\mathrm{t}, J=7.2 \mathrm{~Hz}, 3 \mathrm{H}), 1.43-1.50(\mathrm{~m}$, $1 \mathrm{H}), 1.56-1.62(\mathrm{~m}, 1 \mathrm{H}), 2.11-2.14(\mathrm{~m}, 1 \mathrm{H}), 2.23-2.26(\mathrm{~m}, 1 \mathrm{H}), 2.43$ $(\mathrm{s}, 3 \mathrm{H}), 2.48(\mathrm{dd}, J=15.6,5.4 \mathrm{~Hz}, 1 \mathrm{H}), 2.65(\mathrm{dd}, J=15.6,7.2 \mathrm{~Hz}, 1 \mathrm{H})$, $3.96-4.00(\mathrm{~m}, 1 \mathrm{H}), 4.10$ (q, $J=7.2 \mathrm{~Hz}, 2 \mathrm{H}), 4.67$ (dd, $J=12.0,1.6 \mathrm{~Hz}$, $1 \mathrm{H}), 4.76-4.81(\mathrm{~m}, 1 \mathrm{H}), 7.16-7.18(\mathrm{~m}, 1 \mathrm{H}), 7.22-7.27(\mathrm{~m}, 2 \mathrm{H}), 7.34$ $(\mathrm{d}, J=7.8 \mathrm{~Hz}, 2 \mathrm{H}), 7.46(\mathrm{~d}, J=7.8 \mathrm{~Hz}, 1 \mathrm{H}), 7.80(\mathrm{~d}, J=7.8 \mathrm{~Hz}, 2 \mathrm{H})$.

${ }^{13} \mathrm{C}$ NMR $\left(150 \mathrm{MHz}, \mathrm{CDCl}_{3}\right): \delta=14.4,14.5,21.6,21.9,35.6,37.6,37.7$, $39.7,41.1,41.3,60.7,60.8,68.3,68.8,71.7,71.9,76.9,78.1,127.8(4 C)$, 127.9 (4C), 130.2, 134.5, 145.0, 170.9 .

IR (KBr, neat): 2926, 2853, 1738, 1, 1598, 1474, 1362, 1189, 1097, 814, $793 \mathrm{~cm}^{-1}$.

HRMS (ESI): $m / z[\mathrm{M}+\mathrm{H}]^{+}$calcd. for $\mathrm{C}_{22} \mathrm{H}_{26} \mathrm{ClO}_{6} \mathrm{~S}: 453.1133$; found: 453.1117.

Methyl 4-(( $\left.2 S^{*}, 4 R^{*}, 6 R^{*}\right)$-6-(2-Ethoxy-2-oxoethyl)-4-(tosyloxy)-tetrahydro-2H-pyran-2-yl)benzoate (61, Diastereomeric Mixture; 96:4)

Yield: $305 \mathrm{mg}$ (64\%); colorless oil.

${ }^{1} \mathrm{H}$ NMR $\left(600 \mathrm{MHz}, \mathrm{CDCl}_{3}\right): \delta=1.23(\mathrm{t}, J=7.2 \mathrm{~Hz}, 3 \mathrm{H}), 1.50-1.70(\mathrm{~m}$, $2 \mathrm{H}), 1.98-2.10(\mathrm{~m}, 1 \mathrm{H}), 2.24-2.27(\mathrm{~m}, 1 \mathrm{H}), 2.33-2.36(\mathrm{~m}, 1 \mathrm{H}), 2.46$ (s, $3 \mathrm{H}), 2.63-2.67(\mathrm{~m}, 1 \mathrm{H}), 3.90(\mathrm{~s}, 3 \mathrm{H}), 4.07-4.13(\mathrm{~m}, 3 \mathrm{H}), 4.41(\mathrm{~d}$, $J=10.8 \mathrm{~Hz}, 2 \mathrm{H}), 4.70-4.80(\mathrm{~m}, 1 \mathrm{H}), 7.34(\mathrm{t}, J=16.2 \mathrm{~Hz}, 4 \mathrm{H}), 7.80(\mathrm{~d}$, $J=9.0 \mathrm{~Hz}, 2 \mathrm{H}), 7.80(\mathrm{~d}, J=8.4,2 \mathrm{H})$.

${ }^{13} \mathrm{C}$ NMR $\left(150 \mathrm{MHz}, \mathrm{CDCl}_{3}\right): \delta=14.3,14.4,21.9,37.4,39.7,41.0,61.0$, 72.1, 76.7, 77.1, 125.6, 125.8, 127.8, 129.8, 130.1, 134.3, 145.1, 145.2, 167.0, 170.6 .

IR (KBr, neat): 2938, 2861, 1734, 1626, 1594, 1372, 1161, 1012, 842, $756,672,559 \mathrm{~cm}^{-1}$.

HRMS (ESI): $m / z[M+H]^{+}$calcd. for $\mathrm{C}_{24} \mathrm{H}_{29} \mathrm{O}_{8} \mathrm{~S}$ : 477.1578; found: 477.1594.

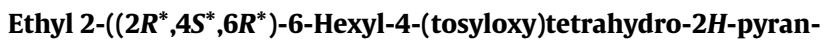
2-yl)acetate (6m, Diastereomeric Mixture; 82:18)

Yield: $223 \mathrm{mg}$ (54\%); yellow oil.

${ }^{1} \mathrm{H}$ NMR $\left(600 \mathrm{MHz}, \mathrm{CDCl}_{3}\right): \delta=0.87(\mathrm{t}, J=7.2 \mathrm{~Hz}, 6 \mathrm{H}), 1.22-1.42(\mathrm{~m}$, $12 \mathrm{H}), 1.91-1.96(\mathrm{~m}, 2 \mathrm{H}), 2.35(\mathrm{dd}, J=15.0,4.8 \mathrm{~Hz}, 1 \mathrm{H}), 2.45(\mathrm{~s}, 3 \mathrm{H})$, $4.52(\mathrm{dd}, J=15.0,8.4 \mathrm{~Hz}, 1 \mathrm{H}), 3.22-3.27(\mathrm{~m}, 1 \mathrm{H}), 3.68-3.72(\mathrm{~m}, 1 \mathrm{H})$, 4.13-4.17 (m, $2 \mathrm{H}), 4.59-4.64(\mathrm{~m}, 1 \mathrm{H}), 7.35$ (d, $J=8.4 \mathrm{~Hz}, 2 \mathrm{H}), 7.79$ $(\mathrm{d}, J=8.4 \mathrm{~Hz}, 2 \mathrm{H})$.

${ }^{13} \mathrm{C}$ NMR $\left(150 \mathrm{MHz}, \mathrm{CDCl}_{3}\right): \delta=14.1,14.2,14.3,21.7,22.6,25.4,31.8$, 35.7, 37.7, 38.0, 41.1, 60.5, 60.6, 60.7, 70.7, 71.8, 71.9, 75.5, 78.2, $125.1,127.7,128.8,129.9,134.4,144.8,170.7$.

IR (KBr, neat): 2925, 2850, 1735, 1640, 1443, 1379, 1177, 1033, 952, $813,668,508 \mathrm{~cm}^{-1}$.

HRMS (ESI): $m / z[\mathrm{M}+\mathrm{H}]^{+}$calcd. for $\mathrm{C}_{22} \mathrm{H}_{35} \mathrm{O}_{6} \mathrm{~S}$ : 427.2149; found: 427.2140. 


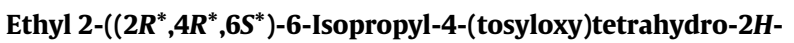
pyran-2-yl)acetate (6n, Diastereomeric Mixture; 93:7)

Yield: $207 \mathrm{mg}$ (52\%); orange gum.

${ }^{1} \mathrm{H}$ NMR (400 MHz, $\left.\mathrm{CDCl}_{3}\right): \delta=0.83(\mathrm{~d}, J=6.6 \mathrm{~Hz}, 3 \mathrm{H}), 0.85(\mathrm{~d}, J=$ $6.6 \mathrm{~Hz}, 3 \mathrm{H}), 1.10-1.14(\mathrm{~m}, 1 \mathrm{H}), 1.23(\mathrm{t}, J=7.2 \mathrm{~Hz}, 3 \mathrm{H}), 1.34-1.44(\mathrm{~m}$, $2 \mathrm{H}), 1.45-1.48$ ( $\mathrm{m}, 1 \mathrm{H}), 1.68-1.72(\mathrm{~m}, 2 \mathrm{H}), 1.88-1.96$ (m, $2 \mathrm{H}), 2.35$ (dd, $J=15.0,4.8 \mathrm{~Hz}, 1 \mathrm{H}), 2.46(\mathrm{~s}, 3 \mathrm{H}), 2.51(\mathrm{dd}, J=15.0,8.4 \mathrm{~Hz}, 1 \mathrm{H})$, 3.31-3.35 (m, $1 \mathrm{H}), 3.68-3.73(\mathrm{~m}, 1 \mathrm{H}), 4.08-4.16(\mathrm{~m}, 2 \mathrm{H}), 4.57-4.66$ $(\mathrm{m}, 1 \mathrm{H}), 7.35(\mathrm{~d}, J=7.8 \mathrm{~Hz}, 2 \mathrm{H}), 7.79(\mathrm{~d}, J=7.8 \mathrm{~Hz}, 2 \mathrm{H})$.

${ }^{13} \mathrm{C}$ NMR $\left(150 \mathrm{MHz}, \mathrm{CDCl}_{3}\right): \delta=14.4,21.9,22.1,23.3,24.6,37.8,38.6$, $41.3,44.8,60.8,72.1,73.8,78.3,127.8,130.1,134.5,145.0,170.9$.

IR (KBr, neat): 2955, 2869, 1738, 1598, 1367, 1190, 1097, 935, 844, $793,669,579 \mathrm{~cm}^{-1}$.

HRMS (ESI): $m / z[M+H]^{+}$calcd. for $\mathrm{C}_{20} \mathrm{H}_{32} \mathrm{O}_{6} \mathrm{~S}$ : 399.1836; found: 399.1820 .

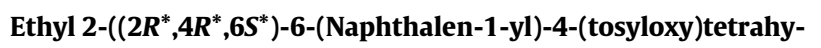
dro-2H-pyran-2-yl)acetate (6p, Diastereomeric Mixture; 95:5)

Yield: $330 \mathrm{mg}$ (64\%); pale-yellow oil.

${ }^{1} \mathrm{H}$ NMR $\left(400 \mathrm{MHz}, \mathrm{CDCl}_{3}\right): \delta=1.21(\mathrm{t}, J=7.0 \mathrm{~Hz}, 3 \mathrm{H}), 1.56-1.66(\mathrm{~m}$, $1 \mathrm{H}), 1.75-1.84(\mathrm{~m}, 1 \mathrm{H}), 2.09-2.15$ ( $\mathrm{m}, 1 \mathrm{H}), 2.28-2.33$ (m, $1 \mathrm{H}), 2.42$ $(\mathrm{s}, 3 \mathrm{H}), 2.48(\mathrm{dd}, J=15.6,5.4 \mathrm{~Hz}, 1 \mathrm{H}), 2.68(\mathrm{dd}, J=15.6,7.4 \mathrm{~Hz}, 1 \mathrm{H})$, 3.96-4.03 (m, $1 \mathrm{H}), 4.11(\mathrm{q}, J=7.0 \mathrm{~Hz}, 2 \mathrm{H}), 4.52(\mathrm{~d}, J=10.2 \mathrm{~Hz}, 1 \mathrm{H})$, 4.79-4.87 (m, $1 \mathrm{H}), 7.31-7.33$ ( $\mathrm{m}, 2 \mathrm{H}), 7.36-7.39$ ( $\mathrm{m}, 1 \mathrm{H}), 7.43-7.45$ (m, $2 \mathrm{H}), 7.71(\mathrm{~s}, 1 \mathrm{H}), 7.77-7.82(\mathrm{~m}, 5 \mathrm{H})$.

${ }^{13} \mathrm{C}$ NMR $\left(100 \mathrm{MHz}, \mathrm{CDCl}_{3}\right): \delta=14.3(2 \mathrm{C}), 21.8,37.6,39.6,41.0,60.8$, 72.4, 78.0, 124.1, 124.7, 126.1, 127.8, 128.1, 128.3, 130.1, 133.1, 133.3, $134.3,138.2,145.0,170.7$.

IR (KBr, neat): 2917, 2845, 1732, 1593, 1357, 1188, 1060, 955, 816, $744,660,552 \mathrm{~cm}^{-1}$.

HRMS (ESI): $m / z[M+H]^{+}$calcd. for $\mathrm{C}_{26} \mathrm{H}_{30} \mathrm{O}_{6} \mathrm{~S}$ : 469.1679; found: 469.1691.

Ethyl 2-((2R*, $\left.4 S^{*}, 6 R^{*}\right)-6$-Benzyl-4-(tosyloxy)tetrahydro-2H-pyran2-yl)acetate (6q, Diastereomeric Mixture; 100:0)

Yield: $233 \mathrm{mg}$ (54\%); pale-yellow oil.

${ }^{1} \mathrm{H} \mathrm{NMR}\left(600 \mathrm{MHz}, \mathrm{CDCl}_{3}\right): \delta=1.23(\mathrm{t}, J=7.2 \mathrm{~Hz}, 3 \mathrm{H}), 1.50-1.70(\mathrm{~m}$, $2 \mathrm{H}), 1.98-2.10(\mathrm{~m}, 1 \mathrm{H}), 2.24-2.27(\mathrm{~m}, 1 \mathrm{H}), 2.33-2.36(\mathrm{~m}, 1 \mathrm{H}), 2.46$ (s, $3 \mathrm{H}), 2.63-2.67(\mathrm{~m}, 1 \mathrm{H}), 4.07-4.13(\mathrm{~m}, 3 \mathrm{H}), 4.41(\mathrm{~d}, J=10.8 \mathrm{~Hz}$, $2 \mathrm{H}), 4.70-4.80(\mathrm{~m}, 1 \mathrm{H}), 7.34(\mathrm{t}, J=16.2 \mathrm{~Hz}, 4 \mathrm{H}), 7.80$ (d, $J=9.0 \mathrm{~Hz}$, $2 \mathrm{H}), 7.80(\mathrm{~m}, 3 \mathrm{H})$.

${ }^{13} \mathrm{C}$ NMR $\left(150 \mathrm{MHz}, \mathrm{CDCl}_{3}\right): \delta=14.3,21.8,37.5,37.6,41.2,42.2,60.8$, 72.1, 76.3, 78.1, 126.5, 127.8 (2C), 128.4, 129.5, 129.6, 130.1, 134.5, $137.8,145.0,170.7$.

IR (KBr, neat): 2928, 2853, 1732, 1590, 1363, 1188, 1082, 932, 838, $749,666,555 \mathrm{~cm}^{-1}$.

HRMS (ESI): $m / z$ [M $+\mathrm{H}]^{+}$calcd. for $\mathrm{C}_{23} \mathrm{H}_{30} \mathrm{O}_{6} \mathrm{~S}$ : 433.1679; found: 433.1693.

\section{Synthesis of 4-Iodotetrahydropyrans; Typical Procedure}

Substrate 6a $(210 \mathrm{mg}, 0.61 \mathrm{mmol})$ and $\mathrm{CeCl}_{3} \cdot 7 \mathrm{H}_{2} \mathrm{O}(270 \mathrm{mg}, 0.73$ $\mathrm{mmol})$ in acetonitrile $(2-5 \mathrm{~mL})$ were treated with NaI $(108 \mathrm{mg}, 0.73$ $\mathrm{mmol}$ ) at $85^{\circ} \mathrm{C}$. The progress of the reaction was monitored by TLC until all starting materials were consumed. Upon completion, the reaction was quenched with dil. $\mathrm{HCl}$, washed with brine and extracted (EtOAc, $2 \times 10 \mathrm{~mL}$ ). The combined organic extracts were dried over anhydrous $\mathrm{Na}_{2} \mathrm{SO}_{4}$, filtered and concentrated under reduced pressure. The residue was further purified by column chromatograph, eluting with EtOAc/hexane; 5:95, to give $\mathbf{8 a}$.

\section{Ethyl 2-((2S*,4S*)-4-Iodotetrahydro-2H-pyran-2-yl)acetate (8a)} Yield: $100 \mathrm{mg}$ (55\%); yellow gum.

${ }^{1} \mathrm{H}$ NMR (400 MHz, $\mathrm{CDCl}_{3}$ ): $\delta=1.26(\mathrm{t}, J=7.2 \mathrm{~Hz}, 3 \mathrm{H}), 2.00$ (dd, $J=$ $12.4,12.4 \mathrm{~Hz}, 1 \mathrm{H}), 2.22-2.25$ ( $\mathrm{m}, 2 \mathrm{H}), 2.35-2.42$ (m, $2 \mathrm{H}), 2.50-2.55$ (dd, $J=16.0,7.7 \mathrm{~Hz}, 1 \mathrm{H}), 3.41-3.48(\mathrm{~m}, 1 \mathrm{H}), 3.72-3.78(\mathrm{~m}, 1 \mathrm{H})$, 3.81-3.85 (m, $1 \mathrm{H}), 4.15$ (q, J = 7.2 Hz, $2 \mathrm{H}), 4.22-4.31(\mathrm{~m}, 1 \mathrm{H})$.

${ }^{13} \mathrm{C} \mathrm{NMR}\left(100 \mathrm{MHz}, \mathrm{CDCl}_{3}\right): \delta=14.4,21.3,39.6,41.0,45.2,60.9,69.5$, 75.7, 170.9 .

IR (KBr, neat): 28.53, 27.83, 1735, 1400, 1253, 1194, 1083, 1030, 988, $543 \mathrm{~cm}^{-1}$.

HRMS (ESI): $m / z[M+H]^{+}$calcd for $\mathrm{C}_{9} \mathrm{H}_{16} \mathrm{IO}_{3}$ : 299.0139; found: 299.0147.

Ethyl 2-((2S*,6S*)-6-(4-Chlorophenyl)-4-iodotetrahydro-2Hpyran-2-yl)acetate (8c, Diastereomeric Mixture; 65:35)

Yield: $146 \mathrm{mg}$ (58\%); yellow gum.

${ }^{1} \mathrm{H} \mathrm{NMR}\left(400 \mathrm{MHz}, \mathrm{CDCl}_{3}\right): \delta=1.22-1.27(\mathrm{~m}, 3 \mathrm{H}), 1.65-1.74(\mathrm{~m}, 1 \mathrm{H})$, 1.97-2.22 (m, 2 H), 2.44-2.55 (m, 2 H), 2.61-2.70 (m, $1 \mathrm{H}), 3.95-4.00$ ( $\mathrm{m}, 2 \mathrm{H}$, minor), 4.14 (q, $J=7.0 \mathrm{~Hz}, 2 \mathrm{H}), 4.34-4.38(\mathrm{~m}, 2 \mathrm{H}$, major), 4.39-4.47 (m, $1 \mathrm{H}$, minor), 4.88-4.92 ( $\mathrm{m}, 1 \mathrm{H}$, major), 7.23 (d, $J=$ $8.5 \mathrm{~Hz}, 2 \mathrm{H}), 7.30(\mathrm{~d}, J=8.5 \mathrm{~Hz}, 2 \mathrm{H})$.

${ }^{13} \mathrm{C}$ NMR $\left(100 \mathrm{MHz}, \mathrm{CDCl}_{3}\right): \delta=14.4,14.5,20.4,28.9,39.9,40.8,40.9$, $42.3,44.3,46.7,60.8,60.9,71.2,75.1,75.6,80.0,127.3,127.4,128.6$, $128.7,133.4,133.6,139.6,140.3,170.7,170.9$.

IR (KBr, neat): 3018, 1734, 1400, 1192, 1074, 819, 653, $552 \mathrm{~cm}^{-1}$. HRMS (ESI): $m / z[M+\mathrm{H}]^{+}$calcd for $\mathrm{C}_{15} \mathrm{H}_{19} \mathrm{ClIO}_{3}$ : 409.0062; found: 409.0062.

Ethyl 2-((2S*,4R* $\left.6 S^{*}\right)-6$-(3-Bromophenyl)-4-iodotetrahydro-2Hpyran-2-yl)acetate (8e, Diastereomeric Mixture; 67:33)

Yield: $152 \mathrm{mg}$ (55\%); yellow gum.

${ }^{1} \mathrm{H}$ NMR (400 MHz, $\left.\mathrm{CDCl}_{3}\right): \delta=1.23-1.29(\mathrm{~m}, 3 \mathrm{H}), 1.66-1.75(\mathrm{~m}, 1 \mathrm{H})$, 1.98-2.23 (m, $2 \mathrm{H}$ ), 2.46-2.57 (m, $2 \mathrm{H}$ ), 2.62-2.72 (m, $1 \mathrm{H}), 3.93-3.98$ (m, $2 \mathrm{H}$, minor), 4.15 (q, $J=7.0 \mathrm{~Hz}, 2 \mathrm{H}), 4.34-4.40$ (m, $2 \mathrm{H}$, major), 4.41-4.50 (m, $1 \mathrm{H}$, minor), 4.88-4.92 (m, $1 \mathrm{H}$, major), 7.16-7.22 (m, $2 \mathrm{H}), 7.37-7.41(\mathrm{~m}, 1 \mathrm{H}), 7.48(\mathrm{~d}, J=8.0 \mathrm{~Hz}, 1 \mathrm{H})$.

${ }^{13} \mathrm{C}$ NMR $\left(100 \mathrm{MHz}, \mathrm{CDCl}_{3}\right): \delta=14.4,20.2,28.7,29.9,39.8,40.8,42.4$, 44.3, 46.7, 61.0, 71.2, 75.0, 75.6, 79.8, 122.8, 124.4, 124.6, 129.0, $129.1,130.1,130.2,130.7,131.0,143.3,144.1,170.8,170.9$.

IR (KBr, neat): 3010, 2859, 1734, 1401, 1194, 1070, $783 \mathrm{~cm}^{-1}$. HRMS (ESI): $m / z[M+\mathrm{H}]^{+}$calcd for $\mathrm{C}_{15} \mathrm{H}_{19} \mathrm{BrIO}_{3}: 452.9557$; found: 452.9587 .

\section{Synthesis of Ethyl 2-(Tetrahydro-2H-pyran-2-yl)acetates; Typical Procedure}

To a stirred solution of $\mathbf{6 a}(200 \mathrm{mg}, 0.58 \mathrm{mmol})$ in DMSO $(0.2 \mathrm{M})$ was slowly added $\mathrm{NaBH}_{4}(67 \mathrm{mg}, 1.75 \mathrm{mmol})$. The reaction mixture was stirred at $85^{\circ} \mathrm{C}$ for $6 \mathrm{~h}$ and progress of the reaction was monitored by TLC. After completion, the reaction was quenched with brine and extracted (EtOAc, $2 \times 10 \mathrm{~mL}$ ). The combined organic phases were dried over $\mathrm{Na}_{2} \mathrm{SO}_{4}$, filtered and concentrated under reduced pressure. The residue was purified by column chromatography, eluting with EtOAc/hexane, 5:95 to give $\mathbf{9 a}$. 
Ethyl 2-(Tetrahydro-2H-pyran-2-yl)acetate (9a)

Yield: 0.56 (53\%); colorless liquid.

${ }^{1} \mathrm{H} \mathrm{NMR}\left(400 \mathrm{MHz}, \mathrm{CDCl}_{3}\right): \delta=1.26(\mathrm{t}, J=7.2 \mathrm{~Hz}, 3 \mathrm{H}), 1.28-1.31(\mathrm{~m}$, $2 \mathrm{H}), 1.47-1.56(\mathrm{~m}, 3 \mathrm{H}), 1.62-1.66(\mathrm{~m}, 1 \mathrm{H}), 1.83-1.84(\mathrm{~m}, 1 \mathrm{H}), 2.38$ $(\mathrm{dd}, J=15.0,5.0 \mathrm{~Hz}, 1 \mathrm{H}), 2.50(\mathrm{dd}, J=15.0,8.0 \mathrm{~Hz}, 1 \mathrm{H}), 3.42-3.49(\mathrm{~m}$, $1 \mathrm{H}), 3.71-3.78(\mathrm{~m}, 1 \mathrm{H}), 3.96(\mathrm{dd}, J=1.0,2.0 \mathrm{~Hz}, 1 \mathrm{H}), 4.15(\mathrm{q}, J=$ $7.2 \mathrm{~Hz}, 2 \mathrm{H})$.

${ }^{13} \mathrm{C}$ NMR $\left(100 \mathrm{MHz}, \mathrm{CDCl}_{3}\right): \delta=14.4,23.5,31.7,41.9,60.6,68.7,74.6$, 171.6.

IR (KBr, neat): 2928, 2855, 1739, 1401, 1166, 1090, 1045, $831 \mathrm{~cm}^{-1}$. HRMS (ESI): $m / z[M+H]^{+}$calcd for $\mathrm{C}_{9} \mathrm{H}_{17} \mathrm{O}_{3}: 273.1172$; found: 273.1166.

\section{Ethyl 2-((2R* $\left.6 S^{*}\right)-6-(4-C h l o r o p h e n y l) t e t r a h y d r o-2 H-p y r a n-2-$ yl)acetate (9c)}

Yield: $86 \mathrm{mg}$ (68\%); colorless liquid.

${ }^{1} \mathrm{H}$ NMR $\left(400 \mathrm{MHz}, \mathrm{CDCl}_{3}\right): \delta=1.24(\mathrm{t}, J=7.0 \mathrm{~Hz}, 3 \mathrm{H}), 1.30-1.48(\mathrm{~m}$, $2 \mathrm{H}), 1.68-1.76$ (m, $2 \mathrm{H}), 1.79-1.87$ (m, $1 \mathrm{H}), 1.91-1.98$ (m, $1 \mathrm{H}), 2.47$ $(\mathrm{dd}, J=15.0,5.8 \mathrm{~Hz}, 1 \mathrm{H}), 2.62(\mathrm{dd}, J=15.0,7.2 \mathrm{~Hz}, 1 \mathrm{H}), 3.93-4.00(\mathrm{~m}$, $1 \mathrm{H}), 4.13$ (q, $J=7.0 \mathrm{~Hz}, 2 \mathrm{H}), 4.38$ (dd, $J=12.0,2.2 \mathrm{~Hz}, 1 \mathrm{H}), 7.24-7.29$ (m, $4 \mathrm{H})$.

${ }^{13} \mathrm{C} \mathrm{NMR}\left(100 \mathrm{MHz}, \mathrm{CDCl}_{3}\right): \delta=14.5,23.9,31.0,33.3,42.0,60.6,75.1$, 79.1, 127.3, 128.5, 133.0, 141.8, 171.6.

IR (KBr, neat): 2938, 2859, 1736, 1400, 1249, 1188, 1087, 1045, 831 $\mathrm{cm}^{-1}$.

HRMS (ESI): $m / z[\mathrm{M}+\mathrm{H}]^{+}$calcd for $\mathrm{C}_{15} \mathrm{H}_{20} \mathrm{ClO}_{3}$ : 283.1095; found: 283.1126.

\section{Ethyl 2-((2R* $\left.6 S^{*}\right)-6$-(Naphthalen-2-yl)tetrahydro-2H-pyran-2- yl)acetate (9p)}

Yield: $90 \mathrm{mg}$ (70\%); colorless oil.

${ }^{1} \mathrm{H} \mathrm{NMR}\left(600 \mathrm{MHz}, \mathrm{CDCl}_{3}\right): \delta=1.28(\mathrm{t}, J=7.0 \mathrm{~Hz}, 3 \mathrm{H}), 1.40-1.47(\mathrm{~m}$, $1 \mathrm{H}), 1.57-1.64(\mathrm{~m}, 2 \mathrm{H}), 1.76-1.83(\mathrm{~m}, 2 \mathrm{H}), 1.97-2.04(\mathrm{~m}, 1 \mathrm{H}), 2.55$ $(\mathrm{dd}, J=15.0,6.0 \mathrm{~Hz}, 1 \mathrm{H}), 2.72(\mathrm{dd}, J=15.0,7.2 \mathrm{~Hz}, 1 \mathrm{H}), 4.05-4.08(\mathrm{~m}$, $1 \mathrm{H}), 4.17$ (q, $J=7.0 \mathrm{~Hz}, 2 \mathrm{H}), 4.60(\mathrm{dd}, J=11.2,1.8 \mathrm{~Hz}, 1 \mathrm{H}), 7.46-7.50$ (m, $3 \mathrm{H}), 7.82-7.85(\mathrm{~m}, 4 \mathrm{H})$.

${ }^{13} \mathrm{C}$ NMR $\left(150 \mathrm{MHz}, \mathrm{CDCl}_{3}\right): \delta=14.5,24.1,31.2,33.3,42.1,60.7,75.2$, $79.9,124.4,124.6,125.8,126.1,127.8,128.0,128.2,133.0,133.5$, 140.8, 171.7 .

IR (KBr, neat): 2934, 2856, 1735, 1634, 1398, 1285, 1192, 1084, 1042 , $817,745 \mathrm{~cm}^{-1}$.

HRMS (ESI): $m / z[M+H]^{+}$calcd for $\mathrm{C}_{19} \mathrm{H}_{23} \mathrm{O}_{3}$ : 299.1642; found: 299.1661.

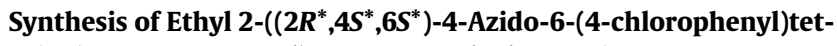
rahydro-2H-pyran-2-yl)acetates; Typical Procedure

A solution of 4-tosyloxy tetrahydropyran $\mathbf{6 c}(190 \mathrm{mg}, 0.43 \mathrm{mmol})$ in anhydrous DMF (3-5 mL) was added to sodium azide ( $85 \mathrm{mg}, 1.3$ mmol) and the mixture was stirred continuously at $100{ }^{\circ} \mathrm{C}$ for $1 \mathrm{~h}$, monitoring by TLC (EtOAc/hexane, 1:9). After completion of the reaction, ice-cold water was added and the mixture was stirred for a further 10-15 minutes. The organic layer was extracted (EtOAc) and the organic extract washed with brine, dried $\left(\mathrm{Na}_{2} \mathrm{SO}_{4}\right)$, filtered and concentrated under reduced pressure. The crude product was purified by column chromatography, eluting with EtOAc/hexane, 10:90, to give 10c.
Yield: $92 \mathrm{mg}$ (66\%); pale-yellow oil.

${ }^{1} \mathrm{H}$ NMR $\left(600 \mathrm{MHz}, \mathrm{CDCl}_{3}\right): \delta=1.27(\mathrm{t}, J=7.2 \mathrm{~Hz}, 3 \mathrm{H}), 1.69-1.72(\mathrm{~m}$, $2 \mathrm{H}), 1.91-1.97$ (m, $2 \mathrm{H}), 2.50$ (dd, $J=15.2,6.0 \mathrm{~Hz}, 1 \mathrm{H}), 2.64$ (dd, $J=$ 15.0, $7.2 \mathrm{~Hz}, 1 \mathrm{H}), 4.15-4.20(\mathrm{~m}, 3 \mathrm{H}), 4.30-4.35(\mathrm{~m}, 1 \mathrm{H}), 4.74(\mathrm{~d}, J=$ $11.2 \mathrm{~Hz}, 1 \mathrm{H}), 7.27(\mathrm{~d}, J=8.4 \mathrm{~Hz}, 2 \mathrm{H}), 7.31(\mathrm{~d}, J=8.4 \mathrm{~Hz}, 2 \mathrm{H})$.

${ }^{13} \mathrm{C}$ NMR $\left(150 \mathrm{MHz}, \mathrm{CDCl}_{3}\right): \delta=14.4,34.5,37.1,41.3,55.9,60.8,69.6$, 73.6, 127.3, 128.7, 133.4, 140.6, 170.9 .

IR (KBr, neat): 2928, 2821, 2105, 1736, 1600, 1400, 1270, 1188, 1066, $1029,836,722 \mathrm{~cm}^{-1}$.

HRMS (ESI): $m / z$ [M $+\mathrm{H}]^{+}$calcd for $\mathrm{C}_{15} \mathrm{H}_{19} \mathrm{ClN}_{3} \mathrm{O}_{3}$ : 324.1109; found: 324.1156 .

Ethyl 2-((2S*,4R*, $\left.6 R^{*}\right)$-4-Azido-6-( $p$-Tolyl)tetrahydro-2H-pyran-2yl)acetate (10h)

Yield: $110 \mathrm{mg}$ (60\%); pale-yellow oil.

${ }^{1} \mathrm{H}$ NMR $\left(400 \mathrm{MHz}, \mathrm{CDCl}_{3}\right): \delta=1.24(\mathrm{t}, J=7.0 \mathrm{~Hz}, 3 \mathrm{H}), 1.62-1.70(\mathrm{~m}$, $2 \mathrm{H}), 1.72-1.79(\mathrm{~m}, 1 \mathrm{H}), 1.87-1.97(\mathrm{~m}, 2 \mathrm{H}), 2.32(\mathrm{~s}, 3 \mathrm{H}), 2.46(\mathrm{dd}, J=$ 15.0, $6.0 \mathrm{~Hz}, 1 \mathrm{H}$ ), 2.63 (dd, $J=15.0,7.0 \mathrm{~Hz}, 1 \mathrm{H}), 4.11-4.18(\mathrm{~m}, 3 \mathrm{H})$, 4.26-4.33 (m, $1 \mathrm{H}), 4.71(\mathrm{~d}, J=10.0 \mathrm{~Hz}, 1 \mathrm{H}), 7.13(\mathrm{~d}, J=8.0 \mathrm{~Hz}, 2 \mathrm{H})$, $7.21(\mathrm{~d}, J=8.0 \mathrm{~Hz}, 2 \mathrm{H})$.

${ }^{13} \mathrm{C}$ NMR $\left(100 \mathrm{MHz}, \mathrm{CDCl}_{3}\right): \delta=14.0,21.3,34.6,36.9,41.4,56.1,60.7$, 69.6, 74.1, 125.9, 129.2, 137.4, 139.1, 171.0.

IR (KBr, neat): 2929, 2823, 2103, 1737, 1620, 1400, 12465, 1187, $1065,1028,809 \mathrm{~cm}^{-1}$.

HRMS (ESI): $m / z[\mathrm{M}+\mathrm{H}]^{+}$calcd for $\mathrm{C}_{16} \mathrm{H}_{22} \mathrm{~N}_{3} \mathrm{O}_{3}$ : 304.1656; found: 304.1700 .

\section{Funding Information}

This work is supported by the Department of Science and Technology (DST), New Delhi (Grant No. EMR/2016/006411), and CoE, MHRD, Government of India.

\section{Acknowledgment}

S.S. gratefully acknowledges the Indian Institute of Technology Guwahati for a Fellowship. The authors are also grateful to the Central Instrument Facility (CIF) of IIT Guwahati for use of the NMR facility.

\section{Supporting Information}

Supporting information for this article is available online at https://doi.org/10.1055/s-0037-1611679.

\section{References}

(1) (a) Polyether Antibiotics, Vols. I and II; Westly, J. W., Ed.; Dekker: New York, 1983. (b) Faulkner, D. J. Nat. Prod. Rep. 2002, 19, 1. (c) Angle, S. R.; El-Said, N. A. J. Am. Chem. Soc. 1999, 121, 10211. (d) Huang, H.; Panek, J. S. J. Am. Chem. Soc. 2000, 122, 9836. (e) Yoshimitsu, T.; Makino, T.; Nagaoka, H. J. Org. Chem. 2004, 69, 1993. (f) Class, Y. J.; DeShong, P. Chem. Rev. 1995, 95, 1843. (g) Norcross, R. D.; Paterson, I. Chem. Rev. 1995, 95, 2041. (h) Nasir, N. M.; Ermanis, K.; Clarke, P. A. Org. Biomol. Chem. 2014, 12, 3323. (i) Cons, B. D.; Bunt, A. J.; Bailey, C. D.; Willis, C. L. Org. Lett. 2013, 15, 2046. 
(2) Wright, A. E.; Botelho, J. C.; Guzman, E.; Harmody, D.; Linley, P.; McCarthy, P. J.; Pitts, T. P.; Pomponi, S. A.; Reed, J. K. J. Nat. Prod. 2007, 70, 412 .

(3) Juch, M.; Rüedi, P. Helv. Chim. Acta 1997, 80, 436.

(4) (a) Kunze, B.; Jansen, R.; Sasse, F.; Hofle, G.; Reichenbach, H. J. Antibiot. 1998, 51, 1075. (b) Kwon, H. J.; Kim, D. H.; Shik, J. S.; Ahn, J. W. J. Microbiol. Biotechnol. 2002, 12, 702.

(5) Tadpetch, K.; Rychnovsky, S. D. Org. Lett. 2008, 10, 4839.

(6) (a) Bondalapati, S.; Reddy, U. C.; Kundu, D. S.; Saikia, A. K. J. Fluorine Chem. 2010, 113, 320. (b) Yadav, J. S.; Reddy, B. V. S.; Anusha, B.; Reddy, U. V. S.; Reddy, V. V. B. Tetrahedron Lett. 2010, 51, 2872. (c) Launay, G. G.; Slawin, A. M. Z.; O'Hagan, D. Beilstein J. Org. Chem. 2010, 6, 41. (d) Yang, J.; Viswanathan, G. S.; Li, C.-J. Tetrahedron Lett. 1999, 40, 1627. (e) Miranda, P. O.; Carballo, R. M.; Martín, V. S.; Padrón, J. I. Org. Lett. 2009, 11, 357.

(7) (a) Yang, X.-F.; Wang, M.; Zhang, Y.; Li, C.-J. Synlett 2005, 12. (b) Reddy, U. C.; Bondalapati, S.; Saikia, A. K. Eur. J. Org. Chem. 2009, 25. (c) Reddy, U. C.; Raju, B. R.; Kumar, E. K. P.; Saikia, A. K.
J. Org. Chem. 2008, 73, 1628. (d) Reddy, B. V. S.; Chaya, D. N.; Yadav, J. S.; Chatterjee, D.; Kunwar, A. C. Tetrahedron Lett. 2011, 52, 2961.

(8) Ahmed, N.; Konduru, N. K. Beilstein J. Org. Chem. 2012, 8, 177.

(9) Yadav, J. S.; Reddy, B. V. S.; Kumar, G. G. K. S. N.; Reddy, G. M. Tetrahedron Lett. 2007, 48, 4903.

(10) Hart, D. J.; Bennett, C. E. Org. Lett. 2003, 5, 1499.

(11) (a) Sultana, S.; Indukuri, K.; Deka, M. J.; Saikia, A. K. J. Org. Chem. 2013, 78, 12182. (b) Gogoi, P.; Das, V. K.; Saikia, A. K. J. Org. Chem. 2014, 79, 8592.

(12) Saikia, A. K.; Indukuri, K.; Das, J. Org. Biomol. Chem. 2014, 12, 7026.

(13) Sabitha, G.; Reddy, K. B.; Reddy, G. S. K. K.; Fatima, N.; Yadav, J. S. Synlett 2005, 2347.

(14) (a) Feuer, H.; Nielsen, A. T. J. Am. Chem. Soc. 1962, 84, 688. (b) McMurry, J. E.; Melton, J. J. Org. Chem. 1973, 38, 4367. 